\title{
The 'Allergy alert' campaign; Use of a Red wristband as an indicator of allergy in all surgical patients of Colombo South Teaching Hospital A quality improvement project
}

\author{
Nimangee Wathsala Mithraratne ${ }^{1^{*}}$, Sita Alwis ${ }^{2}$ \\ Registrar in Anasthesiology ${ }^{1^{*}}$, Consultant Anaesthetist ${ }^{2}$ \\ Colombo South Teaching Hospital, Kalubowila, Sri Lanka.
}

Keywords: allergy alert; anaphylaxis; safety; hospital policy

Editor,

Medical alert wrist bands are widely used in hospitals throughout the world to alert the caregivers on important issues such as allergy, falls risk etc. In many developed countries standardised colours are used in hospital wristbands (e.g.: Red =Allergy) which are clearly identified by any hospital staff member. The national patient safety agency (UK) in 'Standardizing wrist bands improves patient safety: guidance on implementing the Safer Practice Notice 2007' clearly recommends the use of coloured wrist bands to alert on patient safety. ${ }^{1}$

The importance of the use of a wristband for 'Allergy alert' is apparent as the intraoperative incidence of anaphylaxis has been reported at a range from 1 in 4000 to $25,000^{2}$ globally. It is not unusual to overlook a history of allergy leading to severe iatrogenic allergic reactions with resultant morbidity and mortality. Yet, there is no practice to utilize these bands in hospitals of the state sector in Sri Lanka as unfortunately a 'safety check culture' is a rarity in most health care institutions in our country.

\section{Goals}

The aim was to establish the use of a red wrist band in all surgical patients (General surgery and Gynaecology and Obstetrics) with a known allergy when it would be worn throughout their hospital stay. This could potentially aid to minimize all allergic reactions related to non-

*Correspondence: Nimangee Wathsala Mithraratne

E mail: nimangeeis@yahoo.com

https://orcid.org/0000-0002-4421-0099

Received: $31 / 01 / 2018$

Accepted:25/02/2018

DOI: http://doi.org/10.4038/slja.v26i2.8313

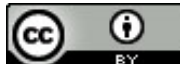

identification of allergy by healthcare staff.

A secondary aim was to make necessary arrangements to establish the 'red band' as a hospital policy to use in all wards (both medical and surgical) and finally to make it a national policy to cover all healthcare institutions.

\section{Design}

The red wrist band had to be low cost, cost efficient and user friendly to patients and staff. Although, ideally it needed to be disposable, due to the cost to the system in provision of a continuous supply of wrist bands and means of disposing them, it was decided that a reusable band to be used that is one inch in width, made of minimally irritable red cotton fabric bearing a 'Velcro' latch.

\section{Pilot Study}

A pilot study was commenced with the help of the hospital administration in ward 23 Colombo South Teaching Hospital. (CSTH) The new change of practice with the intent of improving safety was well received by all staff involved and patients alike.

\section{Implementation and use}

The red band was made a hospital policy to be used in all surgical patients going in to the theatre when indicatedand it was duly supported by the hospital administration with a hospital circular advising the use of it in theatre patients. Notices were used to alert both the patients and hospital staff on the new change.

\section{Further improvement}

Serial audits are a necessity to identify limitations to its use and to assess its benefit. This would help in establishing this in the institution better. Also, such an audit would benefit in bringing about a policy change at institutional and national level to add the use of 'red wrist bands' and other 'risk alert wristbands' to the armamentarium of a 'safety check culture' in Sri Lanka. 


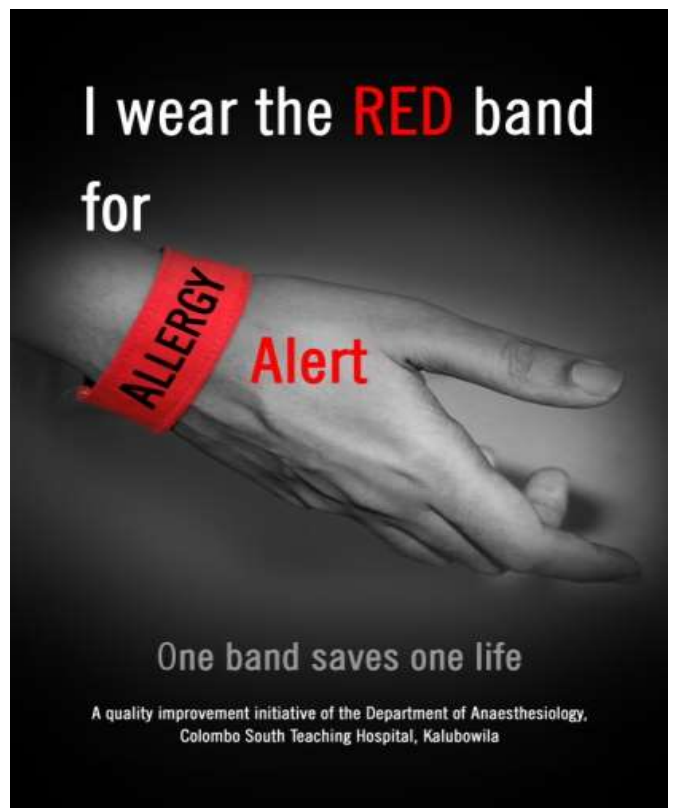

Figure 1: Poster designed for the 'Allergy alert' campaign

\section{References}

1. NPSA. Patient safety resources.

2. Mali S. Anaphylaxis during the perioperative period. Anesth essays Res. 2012;6(2):124-33. https://doi.org/10.4103/0259-1162.108286

PMid:25885604 PMCid:PMC4173455 